\title{
Rediseño de un triciclo de carga pesada, libre de contaminación
}

\section{Redesign of a tricycle to heavy load, pollution-free}

\author{
MORALES-HERNÁNDEZ, Maricela $\dagger^{*}$, VILLALOBOS-NOLASCO, Marco Antonio, DIAZ- \\ SARMIENTO, Bibiana y MINGÜER-ALLEC, Luz María
}

Instituto Tecnológico de Oaxaca / Tecnológico Nacional de México

ID $1^{\mathrm{er}}$ Autor: Maricela, Morales-Hernández / ORC ID: 0000-0002-3521-2041, CVU CONACYT ID: 731036

ID $1{ }^{\mathrm{er}}$ Coautor: Marco Antonio, Villalobos-Nolasco / ORC ID: 0000-0002-7499-8219

ID $2^{\text {do }}$ Coautor: Bibiana, Diaz-Sarmiento / ORC ID: 0000-0003-4350-6311, CVU CONACYT ID: 820776

ID $3^{\text {er }}$ Coautor: Luz María, Mingüer-Allec / ORC ID: 0000-0003-2954-1815, CVU CONACYT ID: 786966

DOI: $10.35429 /$ JOIE.2019.12.3.24.31

Recibido 10 de Septiembre, 2019; Aceptado 10 de Diciembre, 2019

\section{Resumen}

El triciclo de carga es una herramienta ampliamente utilizada en la actualidad debido a su versatilidad, ya que permite transportar de manera efectiva todo tipo de cargas como lo son: materiales de construcción, frutas y verduras, negocios ambulantes, e incluso pasajeros humanos. Actualmente consta de tres ruedas dos al frente y una atrás formando de esa manera un triángulo, sus componentes son: los neumáticos, los rines, el sillín, el montacargas, el cuadro, la cadena de trasmisión, la multiplicación, el piñón trasero, la masa de contrapedal, entre otros. En el presente artículo se describe el rediseño de un triciclo de carga pesada capaz de cargar $700 \mathrm{~kg}$ sin deformar su estructura. Para ello, se ha utilizado el proceso de diseño que propone Budynas y Nisbett en su libro "Diseño en Ingeniería Mecánica de Shigley"; así mismo, se ha realizado el diseño y la simulación en una versión de prueba del software Siemens NX 10 , el cual es un software CAD. Se presentan los planos de todos los componentes diseñados, así como una gráfica de su ensamble.

Triciclo, Rediseño, Carga pesada

\begin{abstract}
The tricycle is a widely used tool nowadays due to its versatility, since it allows effectively transporting all types of loads such as: construction materials, fruits and vegetables, traveling businesses, and even human passengers. Currently consists of three wheels two front and one back forming a triangle, its components are: tires, wheels, seat, lift truck, frame, transmission chain, multiplication, back gear, the counterpedal mass, among others. The article describes the redesign of a heavy load tricycle capable of loading $700 \mathrm{~kg}$ without deforming its structure. For this purpose, it follows the design process proposed by Budynas and Nisbett in his book "Design in Mechanical Engineering of Shigley". Likewise, the design and simulation has been worked in a trial version of the Siemens NX 10 software, which is a CAD software. The plans of all the designed components are presented, as well as a graph of their assembly.
\end{abstract}

Tricycle, Redesign, Heavy load

Citación: MORALES-HERNÁNDEZ, Maricela, VILLALOBOS-NOLASCO, Marco Antonio, DIAZ-SARMIENTO, Bibiana y MINGÜER-ALLEC, Luz María. Rediseño de un triciclo de carga pesada, libre de contaminación. Revista de Ingeniería Innovativa. 2019. 3-12: 24-31

*Correspondencia al Autor (Correo electrónico: moralesh.maricela@gmail.com)

$\dagger$ Investigador contribuyendo como primer Autor. 


\section{Introducción}

De acuerdo con Budynas y Nisbett (2012), diseñar es formular un plan para satisfacer una necesidad específica o resolver un problema, en este caso, el problema que se aborda es la limitante en carga máxima de los triciclos convencionales entre 40 y 240 kilogramos (Trejo, 2014), y además se agrega la característica de que la propulsión de este triciclo es libre de contaminación, ya que se usa solamente el pedaleo, como si fuera una bicicleta convencional, pero que combinando las características de sus componentes se logra un empuje suficiente para llevar un peso de hasta 700 kilogramos.

Si este plan resulta en la creación de algo físicamente real, entonces el producto debe ser funcional, seguro, confiable, competitivo, útil, y es en este momento cuando se puede tomar la decisión de fabricarse y comercializarse (Budynas y Nisbett, 2012).

Existen diferentes aplicaciones del triciclo enfocados a la conservación del ambiente, como lo mencionan Camilo y Veladia (2018) en su tesis. las bicicletas y triciclos aparecen como alternativas vehiculares naturales que podrían solucionar los problemas de emisión y accesibilidad, además de presentar nuevas oportunidades para expandir un nuevo campo de investigación y emprendimiento conocido en Europa como la Ciclologística; esto quiere decir, que las propuestas de utilizar vehículos libres de contaminación es una preocupación general en el mundo actual, de ahí que sea también del interés de los autores del presente trabajo.

Muestra de la preocupación por establecer formalmente y bajo legislaciones el uso del triciclo en diferentes aspectos de la vida diaria, se ha desarrollado en la Unión Europea el programa PRO-E-BIKE (promoting-electricbike-delivery) y se ha generado una mesa de discusión sobre la ciclologística en la ciudad de Bruselas (PRO-E-BIKE, 2015).

Desde 2011, el correo danés utiliza triciclos eléctricos para entregar contenedores de paquetes y paquetes a las empresas; con ello, la necesidad de almacenar los paquetes disminuye sustancialmente (PRO-E-BIKE, 2011).
Por todo lo anterior, el Instituto Tecnológico de Oaxaca no puede mantenerse al margen de estos sucesos y al ser el triciclo una herramienta de trabajo para muchas personas en el municipio de Oaxaca de Juárez, se realiza esta propuesta de diseño de un triciclo de carga pesada. Para lograr lo anterior, y con el fin de desarrollar las características de cada componente del triciclo de carga pesada, se utiliza el software NX 10 fabricado por Siemens, el cual es es una solución integral potente y flexible que ayuda a ofrecer productos mejorados de forma más rápida y eficaz (Siemens, 2019).

Este software CAD (Diseño Asistido por Computadora) /CAM (Manufactura Asistida por Computadora) /CAE (Ingeniería Asistida por Computadora) (Leu, Ghazanfari y Kolan, 2017), ofrece soluciones de diseño, simulación y fabricación con características tecnológicas avanzadas y control de calidad.

Diseño asistido por computadora (CAD) de acuerdo con Villeta (2006) es la utilización de una computadora para poder crear, manipular, analizar y comunicar una idea; proceso que implica desde dibujar planos de productos industriales hasta escribir textos o presupuestos de dichos productos.

El rediseño planteado en este artículo es para un fin específico, usar el triciclo convencional para transportar cargas pesadas cambiando componentes que generan la fuerza en su propulsión, y en caso de requerir transportar mercancías congeladas o frágiles, como lo menciona Arza, Faulin y Serrano (2019), además de tener en cuenta los problemas técnicos, hay que diseñar un modelo apropiado para la optimización de la ruta.

En las secciones que componen el artículo se presenta un rediseño del triciclo para que pueda transportar carga pesada; lo cual sería de gran utilidad en colonias del municipio de Oaxaca de Juárez, ya que muchas de ellas aún no cuentan con la infraestructura para transportación de carga, además se plantea que la propulsión del triciclo siga siendo mediante fuerza de empuje de personas, que es libre de contaminación ya que el mismo ser humano lo manejaría como un triciclo común, el empuje está en el cambio de los elementos que amplifican la fuerza que aplica el pedaleo del mismo. 
Los resultados de la presente investigación beneficiarían a la población de bajos recursos, ya que tendrían un medio de transporte económico y sin contaminación por uso de combustibles fósiles. Por otro lado, la investigación aporta las bases para que se sigan desarrollando otras aplicaciones a este vehículo económico y simple, tal como ya sucede en otros países europeos; y con ello fomentar el uso de energías alternativas para desarrollar actividades cotidianas del ser humano.

El artículo se compone de nueve secciones, en la primera se introduce al lector en el diseño de un triciclo de carga pesada, se explica la idea central del diseño. En la sección dos se plantea la problemática de forma clara, en la siguiente sección se plantea la metodología que se usa en el diseño, en la sección cuatro se describe el desarrollo que se realizó, haciendo uso de un software CAD, se muestran también las diferentes pruebas de esfuerzo que se aplicaron a los componentes del diseño.

La sección cinco muestra los resultados obtenidos, y usando las vistas que proporciona NX 10 se ilustra la forma en que queda el triciclo diseñado con las características planteadas originalmente.

En la sección seis se da el reconocimiento y agradecimiento a las instituciones y personas que aportaron su experiencia y conocimiento en el desarrollo del presente trabajo; en la siguiente sección se dan las conclusiones a las que se llegaron y finalmente se listan las referencias consultadas en la elaboración del presente trabajo.

\section{Planteamiento del problema}

En el municipio de Oaxaca de Juárez, existen 307 colonias (INEGI, 2015), en algunas de ellas la transportación tanto de objetos como de pasajeros humanos, sigue siendo un problema. En los últimos años ha tenido auge el concepto de mototaxi y éstos han proliferado en la ciudad, principalmente en las colonias de los márgenes; sin embargo, los mototaxis también contribuyen a la contaminación del ambiente, ya que utilizan gasolina para su funcionamiento. En contraparte son también populares los triciclos principalmente en la venta ambulante de productos y para transportar algunas cargas poco pesadas.
En el presente trabajo se abordan dos problemáticas: la transportación de carga de objetos pesados y la contaminación del aire. La contaminación se genera cuando se utilizan vehículos que usan combustibles fósiles para la transportación de cargas pesadas como pueden ser: materiales para construcción, víveres, mercancías de uso cotidiano, leña, carbón, etc.; y en lugar del uso de este tipo de vehículos se puede tener triciclos libres de contaminación por combustible, que soporten hasta una carga máxima de 700 kilogramos

\section{Metodología}

Como se menciona en la introducción, se ha tomado como base el proceso de diseño de Budynas y Nisbett (2012). Tal proceso establece seis fases en las que se reconocen múltiples retroalimentaciones e iteraciones, de tal forma, que al final del proceso se alcancen los objetivos y metas planteados para un cierto diseño recopilado de las necesidades reales de los usuarios del triciclo en este caso.

El proceso de diseño se ilustra en la figura 1.

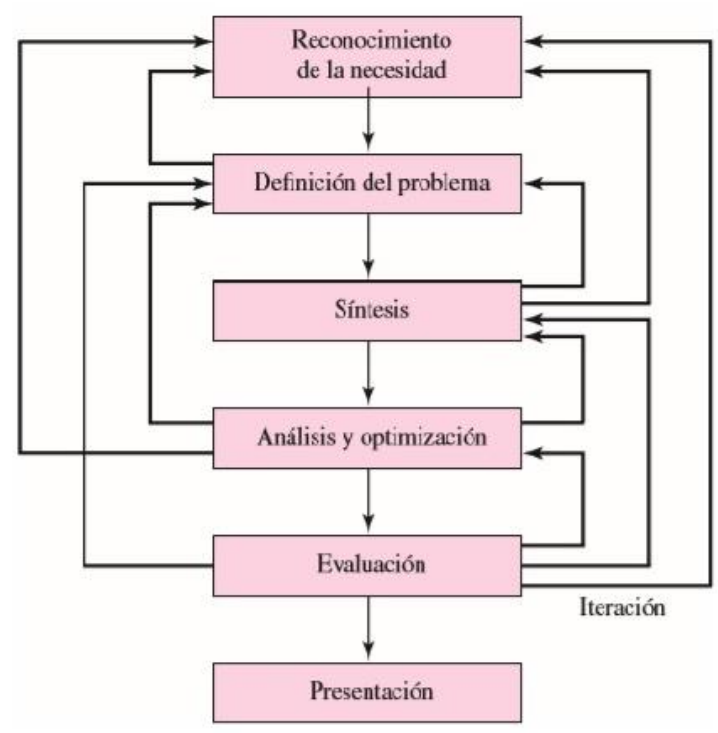

Figura 1 Fases del proceso de diseño Fuente: Budynas y Nisbett, 2008

\section{Desarrollo}

Como se explica en la sección previa, utilizando un modelo de diseño iterativo, se inició el proceso indagando las necesidades de trasportación de carga en las colonias del municipio de Oaxaca de Juárez como son: Estado de Oaxaca, La Joya, Emiliano Zapata, y Montoya. 
De acuerdo con las opiniones de las personas que tienen necesidad de transportar carga, el triciclo de carga perfecto debe ser económico, requerir poco mantenimiento, tener rines fuertes que no se deformen, debe ser fácil de armar y de modificar. Los componentes identificados en el triciclo de carga son los siguientes:

\section{- Neumáticos de caucho \\ - Cámaras internas \\ - $\quad$ Rines \\ - Montacargas \\ - Cuadro del triciclo \\ - Multiplicación \\ - Cadena de transmisión \\ - $\quad$ Piñón trasero}

Con los componentes se realizó una simulación en el programa Siemens NX 10, en la figura 2 se muestra el diseño del neumático y en la tabla 1 se muestra las características del neumático.

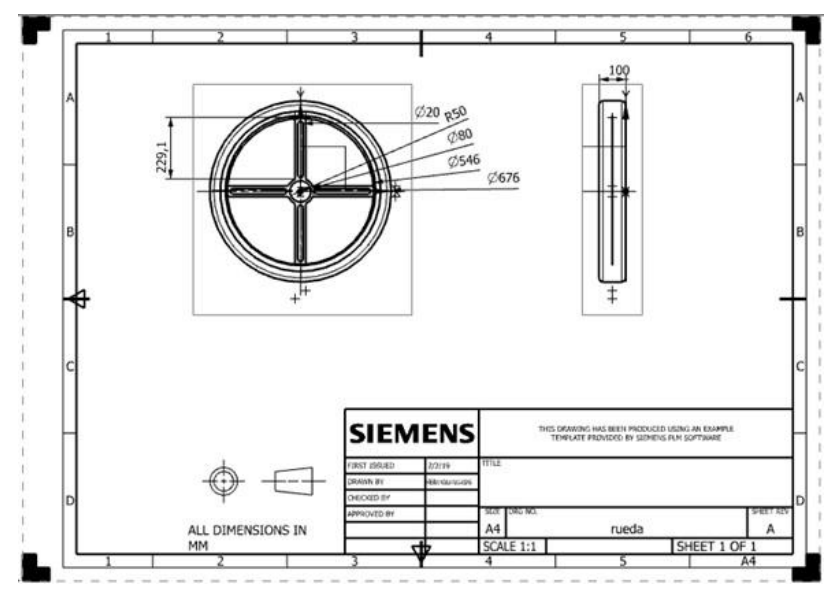

Figura 2 Diseño del neumático

Fuente: Elaboración Propia

\begin{tabular}{|l|l|}
\hline \multicolumn{1}{|c|}{ Nombre } & \multicolumn{1}{|c|}{ Neumático } \\
\hline Descripción & $\begin{array}{l}\text { Serán necesarios tres } \\
\text { neumáticos, dos al frente y uno } \\
\text { atrás. El neumático trasero se } \\
\text { pide con una masa especial para } \\
\text { poder adaptar el piñón trasero, y } \\
\text { los delanteros se acoplan } \\
\text { mediante masas de baleros } \\
\text { sellados }\end{array}$ \\
\hline Ubicación & $\begin{array}{l}\text { Se ubican dos a los costados del } \\
\text { eje de carga y uno se acopla en } \\
\text { la tijera del cuadro trasero }\end{array}$ \\
\hline Material & $\begin{array}{l}\text { Los rines son de acero y las } \\
\text { cubiertas neumáticas son de } \\
\text { motocicleta para poder soportar } \\
\text { el peso }\end{array}$ \\
\hline
\end{tabular}

Tabla 1 Características del neumático

Fuente: Elaboración Propia
En la figura 3 se muestra la simulación del montacargas, mientras en la tabla 2 se muestran las características del mismo.

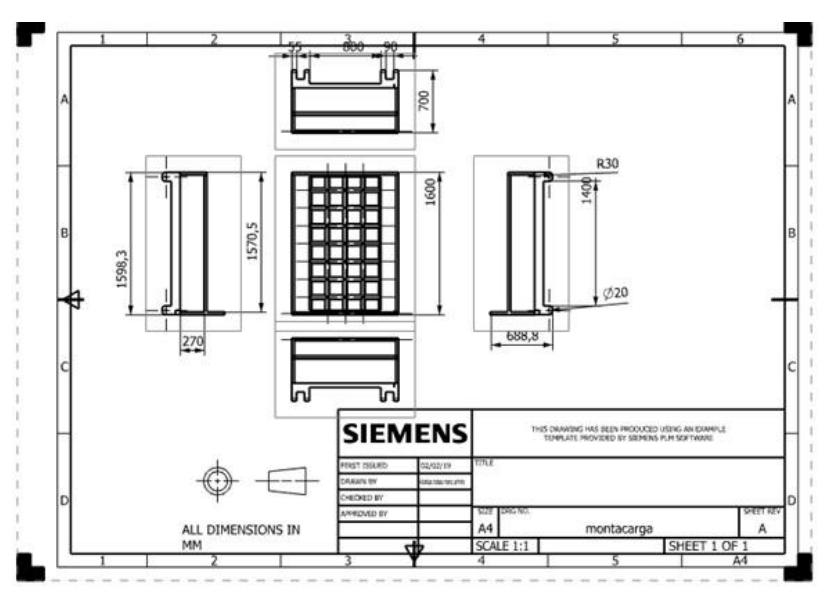

Figura 3 Diseño del montacargas Fuente: Elaboración Propia

\begin{tabular}{|l|l|}
\hline \multicolumn{1}{|c|}{ Nombre } & \multicolumn{1}{c|}{ Montacargas } \\
\hline Descripción & $\begin{array}{l}\text { Se encarga de albergar la carga a } \\
\text { transportar, también funciona como } \\
\text { timón }\end{array}$ \\
\hline Ubicación & $\begin{array}{l}\text { Se acopla a los muelles, al cuadro } \\
\text { del triciclo de carga pesada y se } \\
\text { ubica arriba del eje de carga pesada }\end{array}$ \\
\hline Material & $\begin{array}{l}\text { Está fabricada con acero comercial } \\
\text { o hierro dulce }\end{array}$ \\
\hline
\end{tabular}

Tabla 2 Características del montacargas Fuente: Elaboración Propia

En la figura 4 se presenta el diseño del eje de carga y en la tabla 3 se encuentran las características del eje.

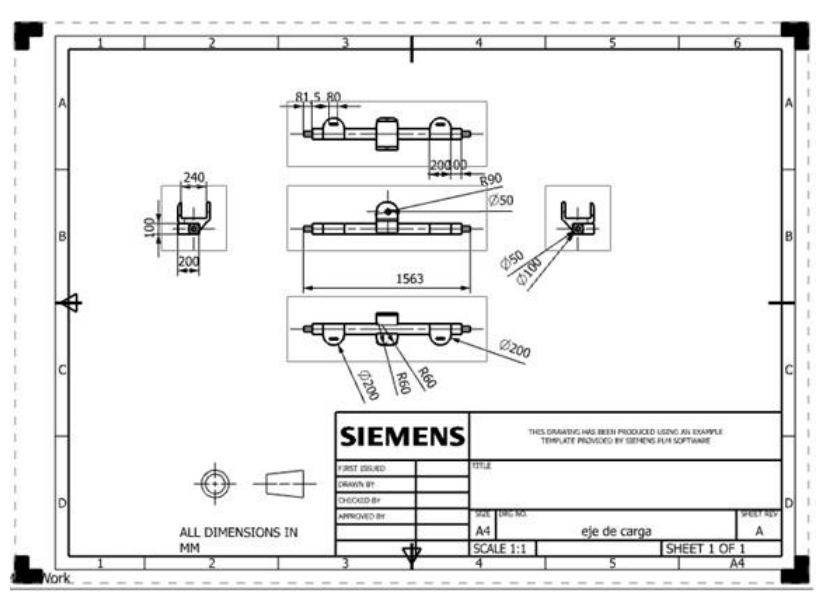

Figura 4 Diseño del eje de carga

Fuente: Elaboración Propia 


\section{Nombre}

\begin{tabular}{|l|l|}
\hline \multicolumn{1}{|c|}{ Nombre } & \multicolumn{1}{|c|}{ Eje de carga } \\
\hline Descripción & $\begin{array}{l}\text { Sobre éste se instalan los } \\
\text { neumáticos delanteros, los } \\
\text { muelles, el monta cargas y se } \\
\text { acopla el cuadro del triciclo de } \\
\text { carga pesada, también controla la } \\
\text { dirección del triciclo. }\end{array}$ \\
\hline Ubicación & $\begin{array}{l}\text { Se aloja en el cuadro del triciclo } \\
\text { de carga pesada en la caja de } \\
\text { centro }\end{array}$ \\
\hline Material & $\begin{array}{l}\text { Está fabricada con acero } \\
\text { comercial o hierro dulce }\end{array}$ \\
\hline
\end{tabular}

Tabla 3 Características del eje de carga

Fuente: Elaboración Propia

En la figura 5 se muestra el diseño del muelle de carga y en la tabla 4 sus características.

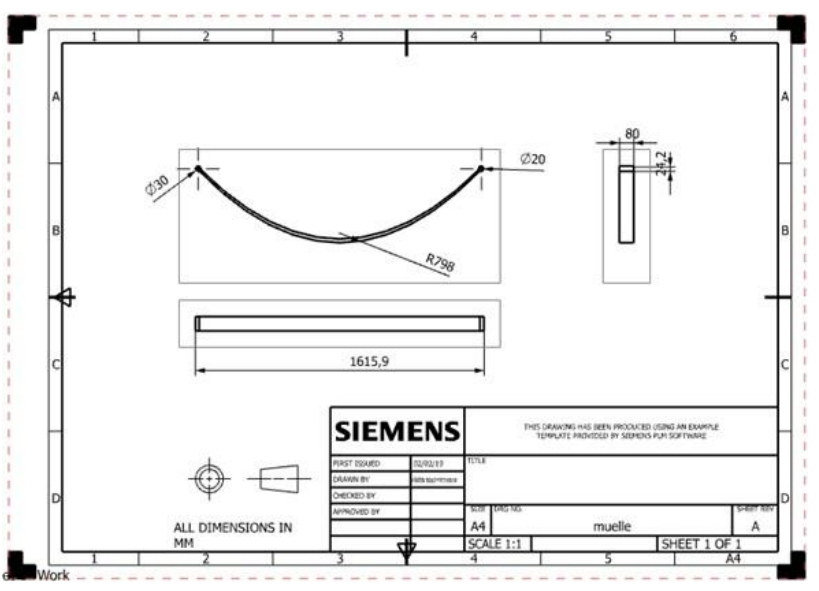

Figura 5 Diseño del muelle de carga Fuente: Elaboración Propia

\begin{tabular}{|l|l|}
\hline \multicolumn{1}{|c|}{ Nombre } & \multicolumn{1}{c|}{ Muelle de carga } \\
\hline Descripción & $\begin{array}{l}\text { Serán necesarios 2, estos se } \\
\text { instalan en el eje de carga y en } \\
\text { el montacargas, su función es } \\
\text { adsorber las irregularidades del } \\
\text { terreno }\end{array}$ \\
\hline Ubicación & $\begin{array}{l}\text { Se ubica entre el eje de carga y } \\
\text { el montacargas }\end{array}$ \\
\hline Material & $\begin{array}{l}\text { Está fabricada con acero } \\
\text { comercial o hierro dulce }\end{array}$ \\
\hline
\end{tabular}

Tabla 4 Características del muelle de carga Fuente: Elaboración Propia

En la figura 6 se presenta el diseño del cuadro del triciclo y en la tabla 5 las características que deseables del mismo.

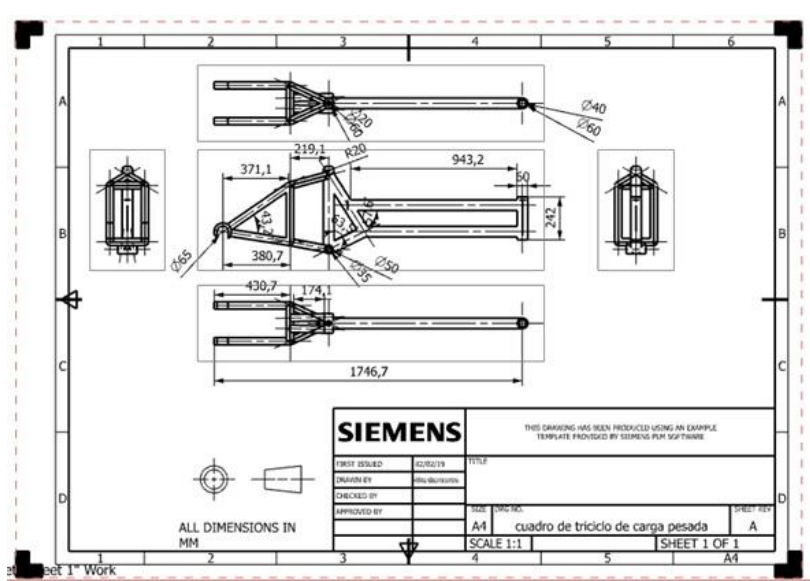

Figura 6 Diseño del cuadro del triciclo Fuente: Elaboración Propia

\begin{tabular}{|l|l|}
\hline \multicolumn{1}{|c|}{ Nombre } & \multicolumn{1}{c|}{ Cuadro } \\
\hline Descripción & $\begin{array}{l}\text { Da soporte a la multiplicación, } \\
\text { rueda trasera, sillín y eje de } \\
\text { carga }\end{array}$ \\
\hline Ubicación & $\begin{array}{l}\text { Se acopla con el eje de carga y } \\
\text { se ubica detrás del monta } \\
\text { cargas. }\end{array}$ \\
\hline Material & Tubos de acero soldados. \\
\hline
\end{tabular}

Tabla 5 Características del cuadro del triciclo Fuente: Elaboración Propia

Los diseños obtenidos en esta etapa se someten a pruebas de esfuerzo de cargas para saber si soportan una carga máxima de 700 kilogramos. Las pruebas de esfuerzo se aplican a los muelles, montacargas, eje de carga y neumáticos. En las figuras 7, 8, 9 y 10 se muestran las pruebas realizadas en las que se utilizó el software de Siemens NX 10.

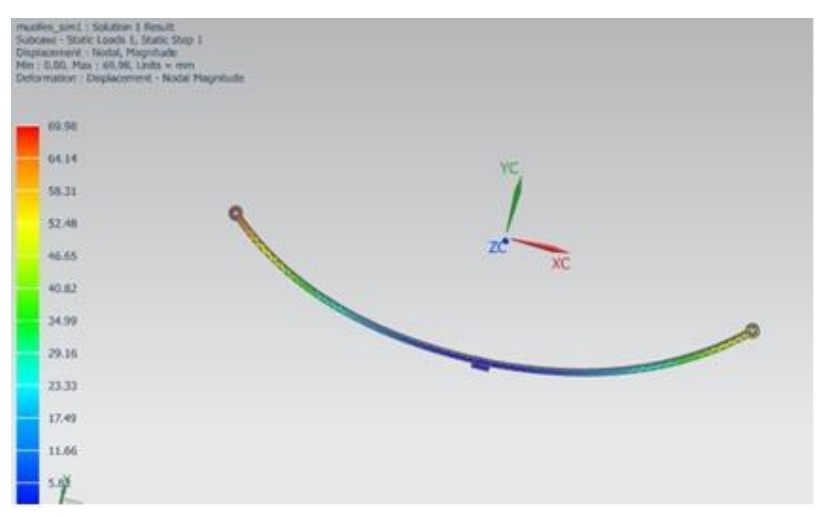

Figura 7 Prueba de esfuerzo en muelles Fuente: Elaboración Propia 


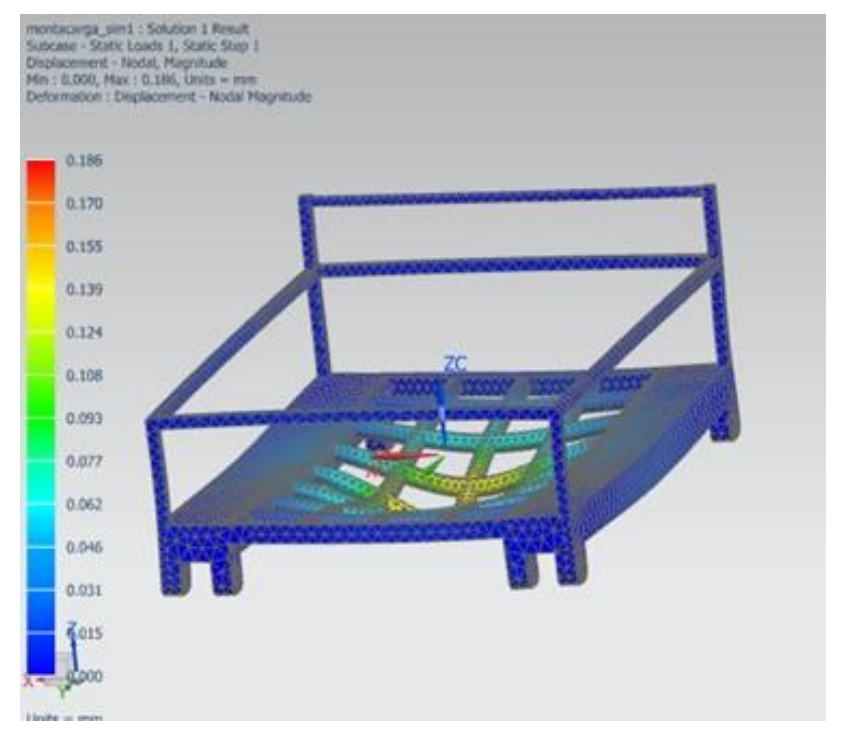

Figura 8 Prueba de esfuerzo en montacargas Fuente: Elaboración Propia

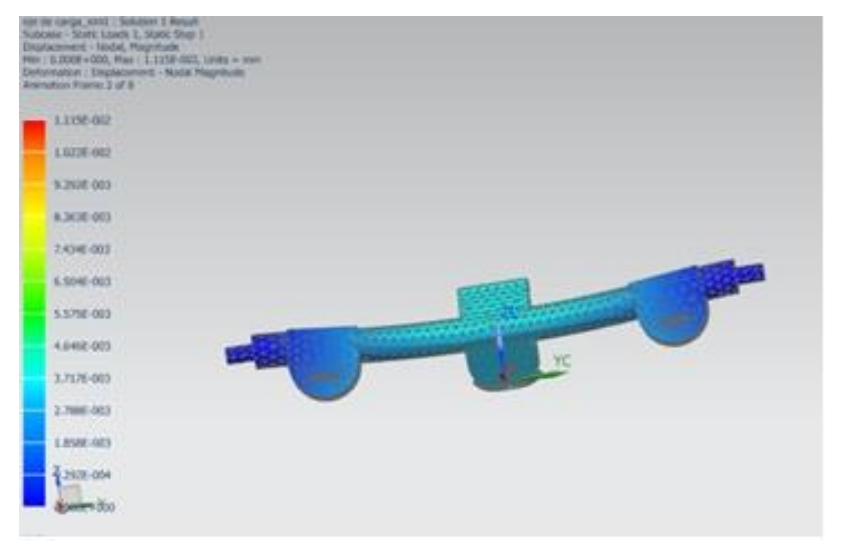

Figura 9 Prueba de esfuerzo en eje de carga Fuente: Elaboración Propia

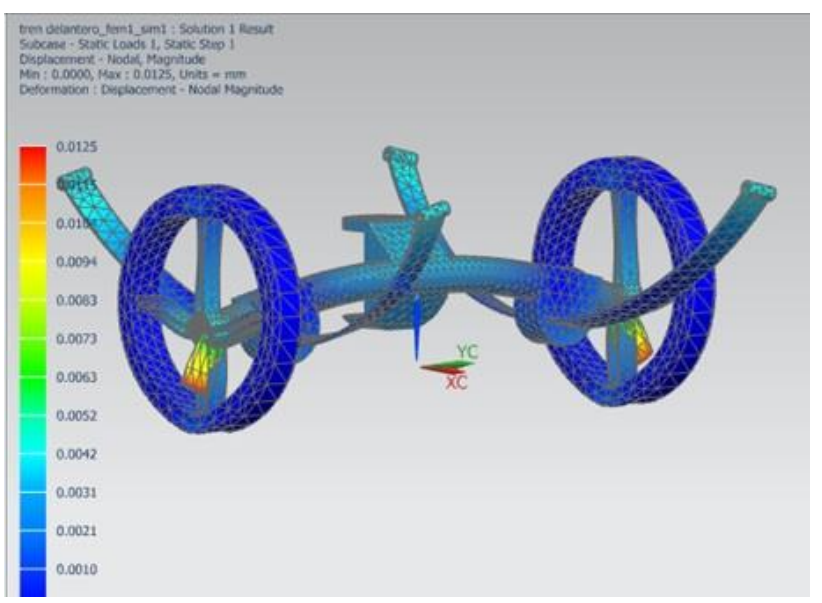

Figura 10 Prueba de esfuerzo en neumáticos Fuente: Elaboración Propia

En todos los casos las deformaciones encontradas son aceptables y no representan un problema para que el triciclo pueda llevar una carga de 700 kilogramos. Las simulaciones han sido de gran utilidad para poder analizar los componentes por separado en una primera instancia y posteriormente todo el triciclo en su conjunto.
Las pruebas a las que se somete el diseño son aceptadas, ya que la deformación que sufre al someterlo a carga es mínima y no afecta el desempeño del mismo.

\section{Resultados}

Después de haber realizado el rediseño del triciclo y de sus componentes; y haber aplicado diferentes pruebas de esfuerzo se obtuvo el diseño definitivo en esta primera fase del trabajo. En la figura 11 se muestra la vista isométrica modelada en Siemens NX 10. En las figuras de la 12 a la 17 se muestran las diferentes vistas del diseño final.

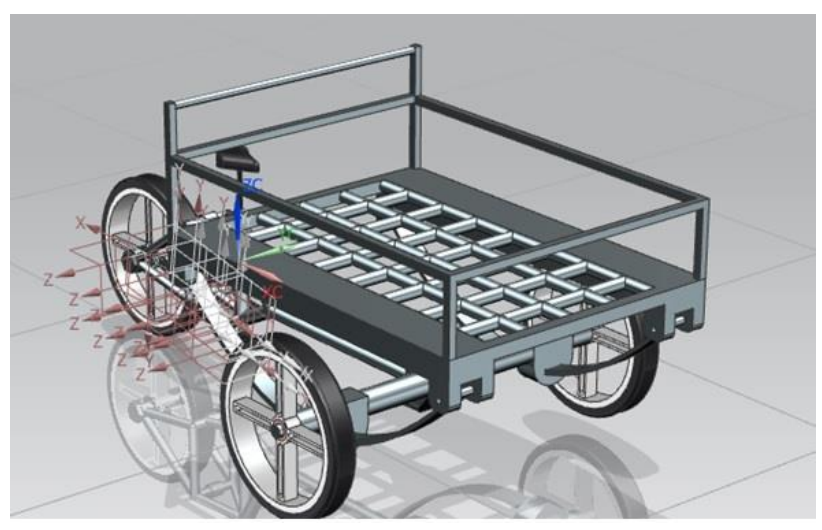

Figura 11 Vista isométrica del triciclo de carga pesada. Fuente: Elaboración Propia

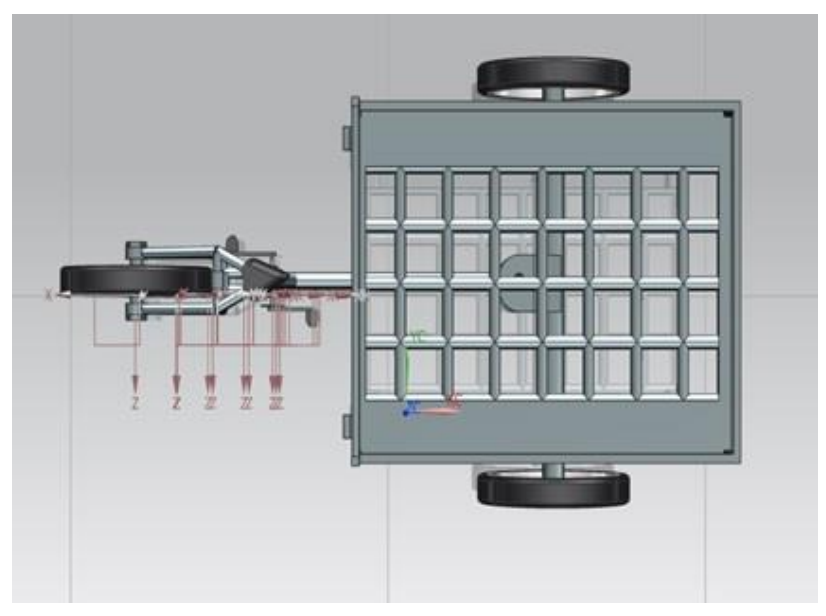

Figura 12 Vista superior del triciclo de carga pesada. Fuente: Elaboración Propia 


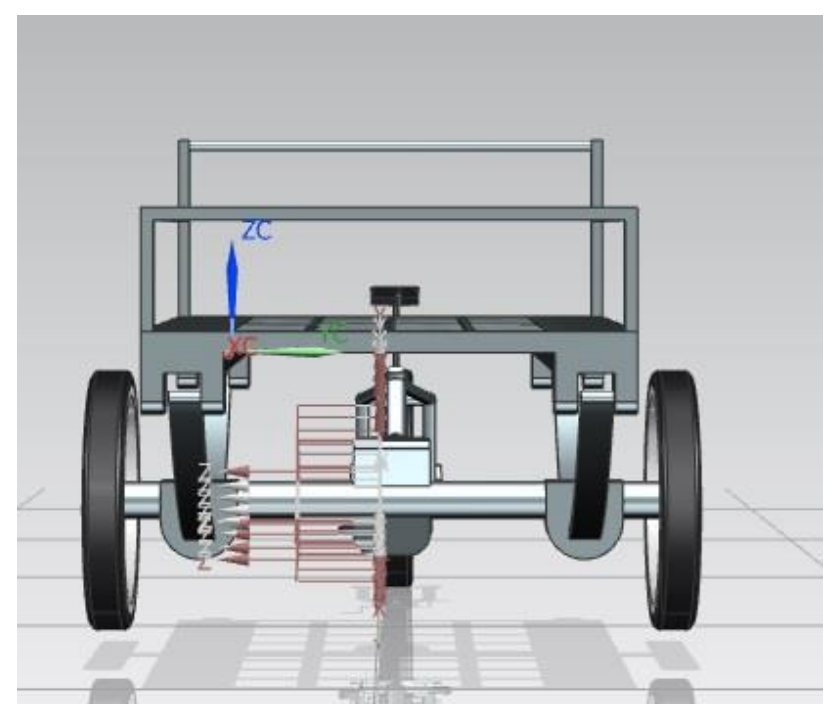

Figura 13 Vista frontal del triciclo de carga pesada Fuente: Elaboración Propia

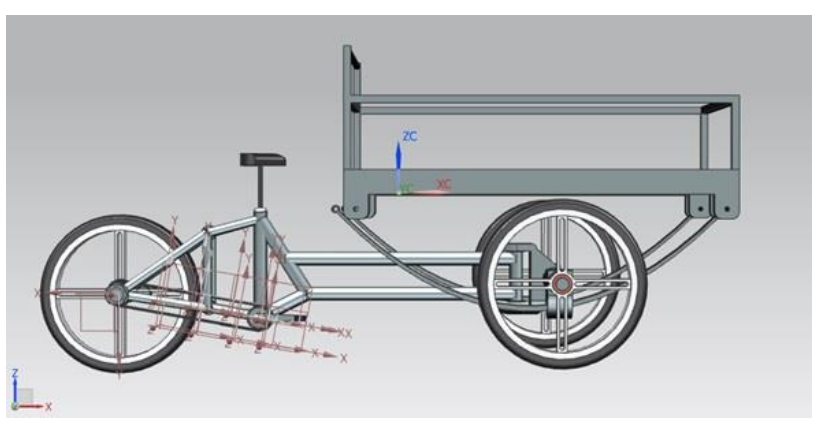

Figura 14 Vista trasera del triciclo de carga pesada Fuente: Elaboración Propia

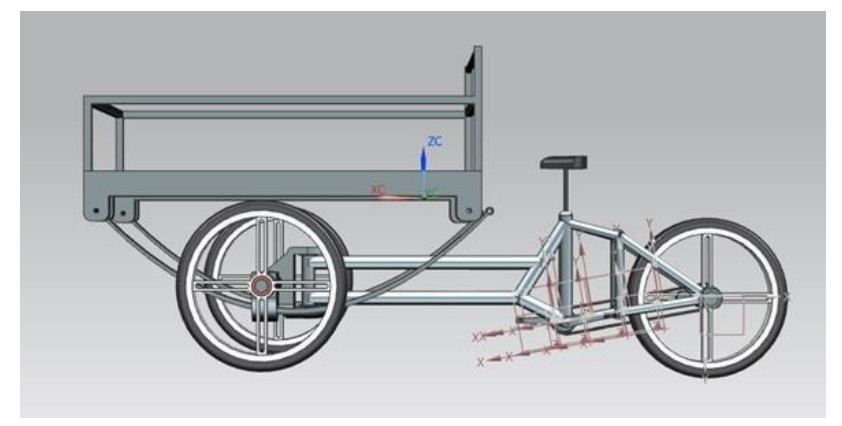

Figura 15 Vista derecha del triciclo de carga pesada Fuente: Elaboración Propia

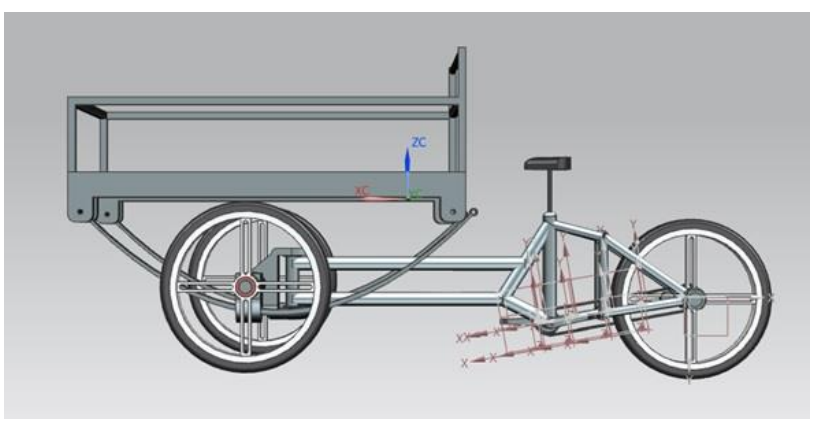

Figura 16 Vista izquierda del triciclo de carga pesada. Fuente: Elaboración Propia

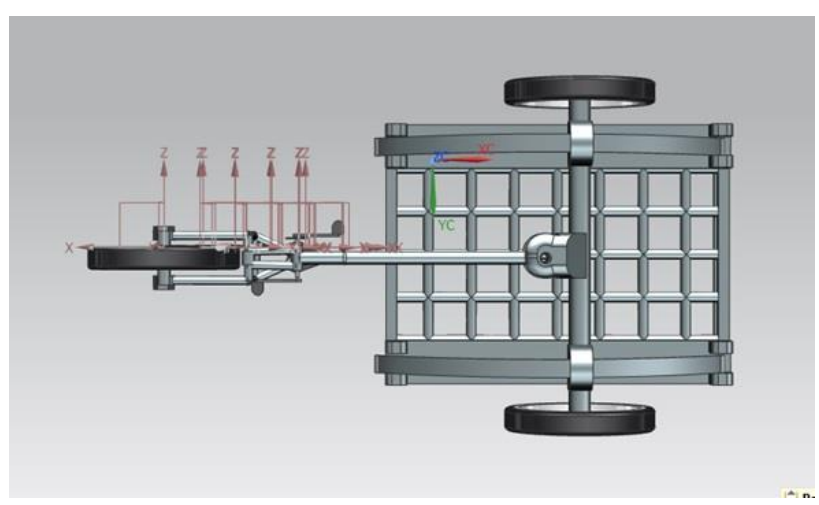

Figura 17 Vista inferior del triciclo de carga pesada Fuente: Elaboración Propia

\section{Agradecimientos}

Se agradece la colaboración y facilidades que se dieron en el desarrollo del presente artículo al Tecnológico Nacional de México, al Instituto Tecnológico de Oaxaca, los profesores investigadores y estudiantes que participaron en el presente trabajo; y que, con su entusiasmo y trabajo aportaron sus mejores ideas al presente documento. Dándole a cada uno los créditos correspondientes.

\section{Conclusiones}

$\mathrm{Al}$ obtener un primer rediseño del triciclo de carga pesada se ha dado un primer paso para posteriormente poder construir un prototipo físico del mismo, para ello, se requiere financiamiento de parte de las instancias correspondientes.

Las especificaciones que se han trabajado en esta primera fase de investigación, arrojan un rediseño con características mejoradas comparado con los triciclos que existen hoy en el mercado; una de las mejoras incorporadas son los neumáticos, ya que estos son de motocicleta con ring ligero. Lo anterior, permite que la deformación de las ruedas por el peso soportado se reduzca favorablemente. Otra mejora incorporada son los baleros sellados, los cuales son un avance en la tecnología de bicicletas ya que estos rodamientos están sellados herméticamente, esta característica impide el paso de partículas de polvo, agua u otros contaminantes que afectan el funcionamiento de los mismos. Y la mejora que es la que se está proponiendo como primicia en este trabajo, la carga que soporta el triciclo, ya que los actuales soportan una carga que oscila entre los 40 y 240 kilogramos aproximadamente; mientras el diseño propuesto en este trabajo puede cargar hasta 700 kilogramos. 
Se comprueba que el uso de tecnologías $\mathrm{CAD}$ es indispensable en el diseño de componentes mecánicos, pues permite revisar antes de invertir en las piezas reales, su comportamiento ante las cargas a las que éstas son sometidas. Así mismo, se hace hincapié en el uso de metodologías aplicadas al diseño mecánico como la que se ha utilizado en este trabajo, que es parecida a otras metodologías que se aplican en otras áreas de la ingeniería, concluyendo que las herramientas durante el desarrollo de cualquier solución a un problema real nos llevan a obtener un trabajo de calidad y con los documentos necesarios para presentarlos a los inversionistas potenciales.

\section{Referencias}

Ángel, C. y Espíndola, V. (2018). Propuesta de distribución de productos alimenticios con bicicletas o triciclos de carga. (tesis de maestría). Universidad Nacional de Colombia, Colombia.

Arza, J., Faulin, J. y Serrano, A. (2019). Análisis de la distribución urbana sostenible de mercancías en el Casco Antiguo de Pamplona (trabajo de fin de grado). España: Universidad Pública de Navarra.

Budynas, R. y Nisbett, J. (2012). Diseño en Ingeniería Mecánica de Shigley. 9a edición. México: McGraw-Hill/Interamericana Editores, S.A. de C.V.

INEGI. (2015). Oaxaca. Visión en cifras. Recuperado de:

https://www.municipiodeoaxaca.gob.mx/upload s/attachment/.../Oaxaca_en_cifras.pdf.

Leu, M., Ghazanfari, A. y Kolan, K. (2017). NX 10 for Engineering Design. Missouri University of Science \& Technology. Recuperado de http://web.mst.edu/ mleu/

PRO-E-BIKE (2011). Buenas prácticas. D 2.1 Current situation analysis. Recuperado de http://www.pro-e-bike.org/buenas-practicas/

PRO-E-BIKE. (23 de noviembre de 2015). Roundtable on Cyclelogistics in Brussels. Recuperado de http://www.pro-ebike.org/2015/11/23/roundtable-on-

cyclelogistics-in-brussels/
Siemens. (2019). Productos. NX para diseño. Recuperado de https://www.plm.automation.siemens.com/glob al/es/products/nx/nx-for-design.html

Trejo. (2014). Triciclos de carga. Recuperado de http://triciclosdecarga.com/triciclo-de-cargacharger-reforzado-R300.php

Villeta, J. (2006). Desarrollo de Proyectos usando CAD. República Dominicana: Universidad de Santo Domingo. 